\section{Recent measurements of soft X-ray emission from the DPF-1000U facility}

\author{
Władysław Surała, \\ Marek J. Sadowski, \\ Marian Paduch, \\ Ewa Zielinska, \\ Krzysztof Tomaszewski
}

\begin{abstract}
Soft X-ray imaging is a very useful diagnostic technique in plasma-focus (PF) experiments. This paper reports results of four experimental sessions which were carried out at the DPF-1000U plasma-focus facility in 2013 and 2014. Over 200 discharges were performed at various experimental conditions. Measurements were taken using two X-ray pinhole cameras with a line of sight perpendicular to the z-axis, at different azimuthal angles (about $20^{\circ}$ and $200^{\circ}$ ), and looking towards the centre of the PF-pinch column. They were equipped with diaphragms $1000 \mu \mathrm{m}$ or $200-300 \mu \mathrm{m}$ in diameter and coated with filters of $500 \mu \mathrm{m} \mathrm{Al}$ foil and $10 \mu \mathrm{m}$ Be foil, respectively. Data on the neutron emission were collected with silver activation counters. For time-resolved measurements the use was made of four PIN diodes equipped with various filters and oriented towards the centre of the PF-column, in the direction perpendicular to the electrode axis. The recorded X-ray images revealed that when the additional gas-puff system is activated during the discharge, the stability of the discharge is improved. The data collected in these experiments confirmed the appearance of a filamentary fine structure in the PF discharges. In the past years the formation of such filaments was observed in many Z-pinch type experiments. Some of the recorded X-ray images have also revealed the appearance of the so-called hot-spots, i.e. small plasma regions of a very intense X-ray emission. Such a phenomenon was observed before in many PF experiments, e.g. in the MAJA-PF device, but it has not been investigated so far in a large facility such as the DPF-1000U. The time-resolved measurements provided the evidence of a time lapse between the X-ray emission from plasma regions located at different distance from the anode surface. The formation of distinct 'hot-spots' in different instants of the DPF-1000U discharge was also observed.
\end{abstract}

Key words: plasma focus $\bullet$ X-ray emission $\bullet$ X-ray images $\bullet$ gas-puffing $\bullet$ filaments $\bullet$ hot-spots

\author{
W. Surała \\ National Centre for Nuclear Research (NCBJ), \\ 7 Andrzeja Soltana Str., 05-400 Otwock/Swierk, Poland, \\ Tel.: +48 22718 0536, Mobile: +48 503731 794, \\ Fax: +48 227793481 , \\ E-mail: Wladyslaw.Surala@ncbj.gov.pl

\section{J. Sadowski} \\ National Centre for Nuclear Research (NCBJ), \\ 7 Andrzeja Soltana Str., 05-400 Otwock/Swierk, Poland \\ and Institute of Plasma Physics and Laser Microfusion \\ (IPPLM), \\ 23 Hery Str., 01-497 Warsaw, Poland \\ M. Paduch, E. Zielinska \\ Institute of Plasma Physics and Laser Microfusion \\ (IPPLM), \\ 23 Hery Str., 01-497 Warsaw, Poland \\ K. Tomaszewski \\ ACS Sp. z o.o., \\ 23 Hery Str., 01-497 Warsaw, Poland
}

Received: 15 June 2014

Accepted: 30 January 2015

\section{Introduction}

Soft X-ray (SXR) imaging is a very useful diagnostic technique in plasma-focus (PF) experiments, since it provides important information on spatial characteristics of the discharge, such as the degree of symmetry, the stability and the plasma density $[1,2]$. Moreover, with this technique it is possible to observe such phenomena as 'hot-spots' and 'filament' appearing in particular during the phase of maximum compression [3].

This paper reports on studies of the soft X-ray emission from the high-temperature plasma generated in a large plasma-focus facility such as the DPF-1000U [4]. Four experimental sessions were carried out in 2013 and 2014. Here we present selected results from recent X-ray measurements obtained with the DPF-1000U machine, which was operated at various experimental conditions, i.e., with and without the additional gas-puffing, with different working gases and at different pressures. 


\section{Experimental setup}

All the reported experiments were carried out using the Mather-type DPF-1000U facility, which is equipped with a tubular copper anode $230 \mathrm{~mm}$ in diameter, with a fast-acting gas valve located at its axis. The outer cathode consists of twelve stainless steel tubes $(80 \mathrm{~mm}$ in diameter) which are distributed symmetrically around the circle $400 \mathrm{~mm}$ in diameter. Both electrodes are $460 \mathrm{~mm}$ long. Discharges were performed mainly with the pure deuterium filling under the initial pressure ranging from 1.2 to 1.8 Torr, while some of them were carried out with the neon filling at 0.6 Torr. In all cases the main condenser bank, which supplied plasma discharges, was charged up to $23 \mathrm{kV}$, which corresponded to the energy of $330 \mathrm{~kJ}$.

The diagnostic setup consisted of two X-ray pinhole cameras for time-integrated measurements, which had input diaphragms $1000 \mu \mathrm{m}$ and 200-300 $\mu \mathrm{m}$ in diameter, coated with filters made of $500 \mu \mathrm{m} \mathrm{Al}$ foil and $10 \mu \mathrm{m}$ Be foil, respectively. The $\mathrm{Al}$ filter transmitted radiation $>17.1 \mathrm{keV}$, while the Be filter transmitted radiation $>1.2 \mathrm{keV}$. Both of the cameras were oriented towards the centre of the PF current sheath during its collapse phase, as shown in Fig. 1.

The pinhole with the Al filter was placed side-on, and the pinhole with the Be filtered was placed either

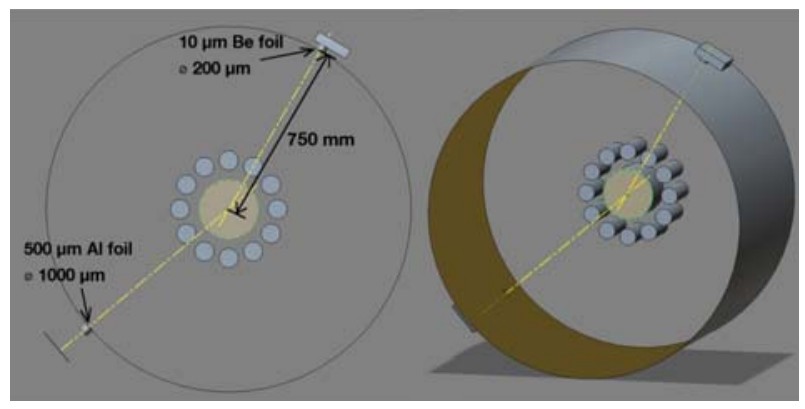

Fig. 1. Positions of the X-ray pinhole cameras with respect to the DPF-1000U electrodes, as seen from the front (left picture) and in perspective (right picture).

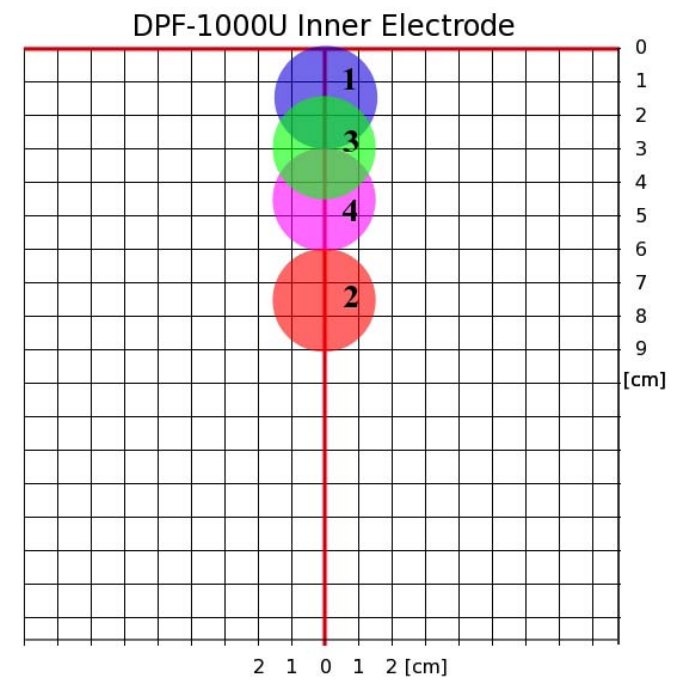

side-on or at an angle of $75^{\circ}$ to the z-axis (images taken at that angle had of course an elliptical shape).

For preliminary time-resolved measurements four PIN diodes equipped with various filters $(10 \mu \mathrm{m} \mathrm{Be}$, $3 \mu \mathrm{m} \mathrm{Al}, 0.6 \mu \mathrm{m} \mathrm{Al}$ with $1.5 \mu \mathrm{m}$ Mylar, and $1 \mu \mathrm{m} \mathrm{Be}$ ) were utilized. Those filters transmitted radiation $>1.2 \mathrm{keV},>1.1 \mathrm{keV},>1 \mathrm{keV}$, and $>0.5 \mathrm{keV}$, respectively.

In the experimental sessions carried out in June 2013 (shots \#9979 - \#10 003) and September 2013 (\#10 062 - \#10 129) four PIN diodes were used, which looked at the point located at a distance of $z=45 \mathrm{~mm}$ from the anode surface. Those PIN diodes were oriented perpendicularly to the axis and they were equipped with different absorption filters. In the sessions carried out in November-December 2013 (shots \#10 134 - \#10 245) and February 2014 (shots \#10 269 - \#10 345) all the applied PIN diodes were equipped with $10 \mu \mathrm{m}$ Be filters and oriented at various points on the z-axis, as shown in Fig. 2. That configuration allowed the space-resolved measurements to be performed.

In these experimental sessions data on the neutron emission were collected by means of silver activation counters. An averaged neutron yield amounted to $10^{10}$ neutrons per shot.

In order to record time-integrated spatial distribution of the X-ray radiation photographic images were recorded with the use of the XBM ${ }^{\circledR}$ X-ray Retina film (during the experimental session in June 2013) and the AA400 Kodak Industrex ${ }^{\circledR}$ film (during subsequent sessions). Each exposed film was developed for $5 \mathrm{~min}$ in a $20^{\circ} \mathrm{C}$ water-solution of a developer. The use was made of the dedicated Kodak Developer and Replenisher. The developed X-ray images had different background intensity due to changes in the X-ray emission, especially in the shots performed with the neon-filling or neon-admixture.

Time-resolved data were obtained from four PIN diodes coupled with a Tektronix ${ }^{\circledR}$ oscilloscope through independent channels. Some shots were performed with the deuterium or neon puffing

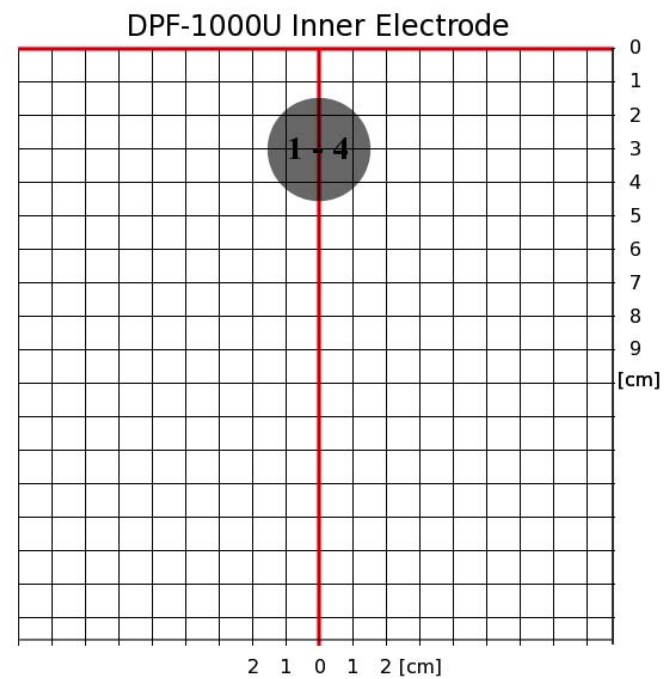

Fig. 2. Two configurations of the PIN diodes for time-resolved X-ray intensity measurements. Vertical line corresponds to the $\mathrm{z}$-axis of the discharge. Small circles on the left picture correspond to different channels described in Table 2. 
Table 1. Experimental data from the DPF-1000U discharges described above

\begin{tabular}{|c|c|c|c|c|c|}
\hline Shot no. & Working gas & $\begin{array}{c}\text { Working pressure } \\
\text { [Torr] }\end{array}$ & Puffed gas & $\begin{array}{c}\text { Neutron yield } \\
{\left[10^{10}\right]}\end{array}$ & Notes \\
\hline 9987 & $\mathrm{D}_{2}$ & 1.8 & $\mathrm{D}_{2}$ & 0.3 & hot-spots \\
\hline 10002 & $\mathrm{D}_{2}$ & 1.8 & none & 4.9 & CFC target visible \\
\hline 10003 & $\mathrm{D}_{2}$ & 1.8 & none & 0.3 & CFC target visible \\
\hline 10074 & $\mathrm{D}_{2}$ & 1.5 & $\mathrm{D}_{2}$ & 7.5 & filaments \\
\hline 10080 & $\mathrm{D}_{2}$ & 1.5 & $\mathrm{D}_{2}$ & 11.0 & filaments \\
\hline 10143 & $\mathrm{D}_{2}$ & 1.5 & $\mathrm{D}_{2}$ & 13.0 & hot-spots \\
\hline 10144 & $\mathrm{D}_{2}$ & 1.5 & none & 1.3 & hot-spots \\
\hline 10187 & $\mathrm{D}_{2}$ & 1.5 & $\mathrm{D}_{2}$ & 16.8 & \\
\hline 10220 & $\mathrm{D}_{2}$ & 1.2 & $\mathrm{D}_{2}$ & 2.8 & \\
\hline 10230 & $\mathrm{D}_{2}$ & 1.2 & none & 3.3 & filaments \\
\hline 10235 & $\mathrm{D}_{2}+\mathrm{Ne}$ & 1.26 & none & 2.6 & $\mathrm{Ne}(0.06$ Torr $)$ \\
\hline 10244 & $\mathrm{D}_{2}+\mathrm{Ne}$ & 1.55 & $\mathrm{D}_{2}$ & 1.3 & Ne (0.05 Torr) \\
\hline 10271 & $\mathrm{D}_{2}$ & 1.2 & none & 0.3 & \\
\hline 10280 & $\mathrm{D}_{2}$ & 1.5 & none & 3.0 & \\
\hline 10295 & $\mathrm{Ne}$ & 0.6 & none & 0.01 & \\
\hline 10329 & $\mathrm{Ne}$ & 0.6 & $\mathrm{D}_{2}$ & 1.6 & \\
\hline 10331 & $\mathrm{D}_{2}$ & 1.5 & $\mathrm{D}_{2}$ & 5.9 & \\
\hline 10333 & $\mathrm{D}_{2}$ & 1.5 & $\mathrm{D}_{2}$ & 3.7 & filaments \\
\hline 10336 & $\mathrm{D}_{2}$ & 1.5 & $\mathrm{D}_{2}$ & 12.5 & \\
\hline 10338 & $\mathrm{D}_{2}$ & 1.5 & $\mathrm{D}_{2}$ & 2.2 & hot-spots \\
\hline 10339 & $\mathrm{D}_{2}$ & 1.5 & $\mathrm{Ne}$ & 0.5 & \\
\hline
\end{tabular}

(about $1 \mathrm{~cm}^{3}$, at the pressure of 1500 Torr) along the anode axis. That gas injection was usually performed about 1.5-2 ms before the main discharge triggering.

During the experiments it was necessary to overcome several experimental difficulties. The geometrical and emission characteristics of the PF discharges varied from shot to shot. Hence, a large number of shots was needed in order to reach unambiguous conclusions. In some cases the soft X-ray emission intensity was so weak that it was impossible to record a high-resolution pinhole image, even though sensitive X-ray films were used (the resolution was limited to $200-300 \mathrm{~nm}$ ). Therefore, in order to obtain

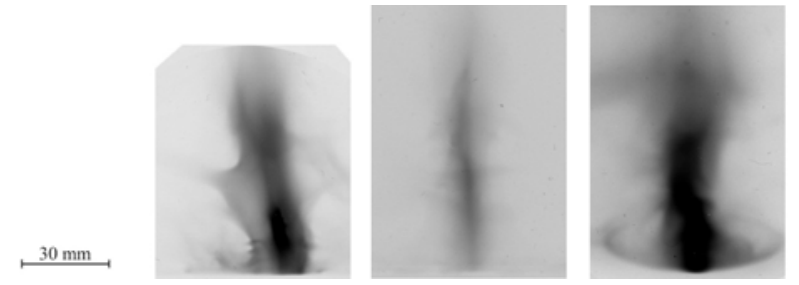

Fig. 3. Discharges without gas-puffing (from left to right), the applied working gas: shot \#10 235 - deuterium with neon admixture, shot \#10280 - pure deuterium, and shot \#10 295 - pure neon.

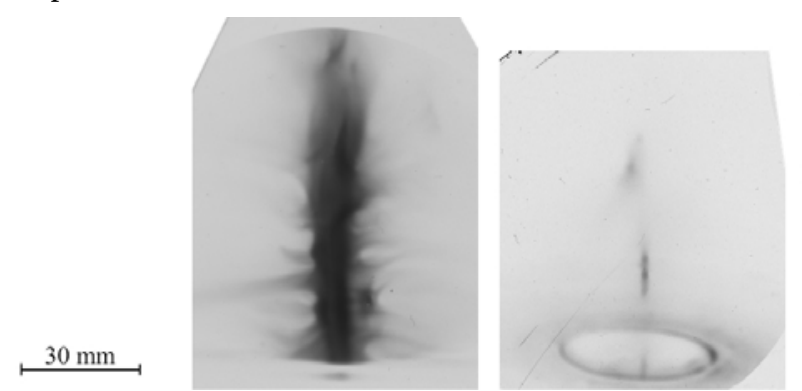

a satisfactory image intensity and image resolution, a proper selection of the pinhole diameter was necessary. The fact that various X-ray films were used (see above) had the consequence that some images had higher background blackening and lower contrast.

\section{Experimental results: time-integrated images}

During the reported DPF-1000U experiments about 200 integrated X-ray images were collected, together with the corresponding time-resolved data (waveforms of signals from the PIN diodes) and neutron yields. The basic information about the performed shots is presented in Table 1. Exemplary images from PF shots performed with and without gas-puffing are presented in Figs. 3 and 4.

A comparison of the recorded X-ray images revealed that the gas-puffing increased the discharge stability, and it proved to be correlated with a higher neutron yield (as recorded with silver activation counters). Almost all PF shots with the deuterium gas-puffing showed a clearly visible axial region of an increased X-ray emission and good axial symmetry. In some cases shots performed without any

Fig. 4. Discharges with the gas-puffing (from left to right): shot \#10 244 - working gas deuterium with a neon admixture and deuterium gas-puffing, shot \#10 329 - working gas neon and deuterium gas-puffing, shot \#10 331 - working gas deuterium and deuterium gas-puffing, shot \#10 339 - working gas neon and neon gas-puffing. 


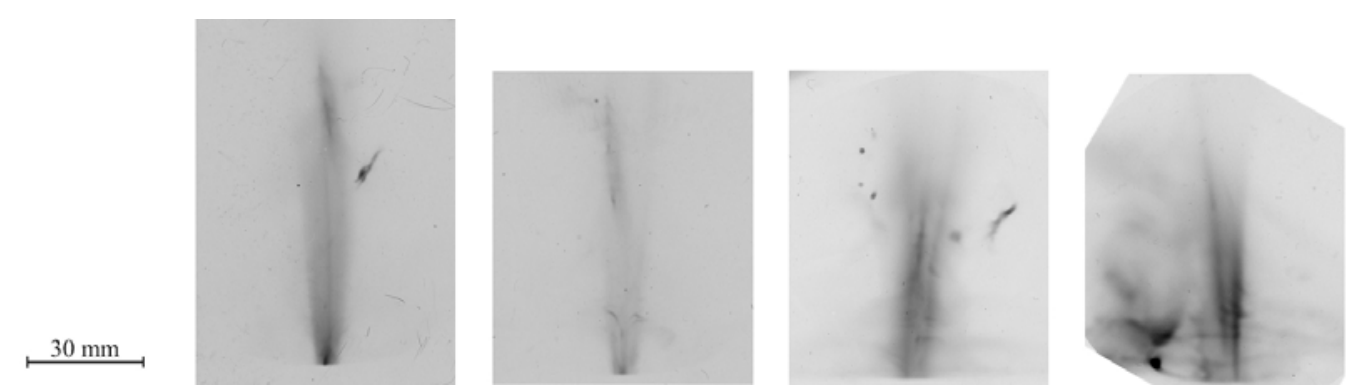

Fig. 5. Discharges with a visible filamentary structure (from left to right): shot \#10 074 - a quasi-axial 'core', shot \#10 080 - filaments collapsing in the front of the anode, shots \#10 230 and \#10 333 - distinct filaments.

gas-puffing also showed an intense X-ray emission, but the images were not axially symmetric and consisted of random blobs of emitting plasma. The use of neon either as a working gas or as an admixture resulted in a strong increase in the X-ray emission. In almost all shots with the deuterium gas-puffing and pure neon as working gas it was found that X-rays are emitted mostly from the injected gas.

The appearance of distinct plasma filaments even during the maximum compression of the PF pinch column was for the first time observed in the POSEIDON facility [3]. In the past years the formation of such filaments has had been observed in many Z-pinch type experiments [5]. The soft X-ray pinhole images obtained during the recent DPF-1000U experiments have also confirmed the existence of a filamentary fine structure, and a quasi-axial 'core' was observed in discharges with the gas-puffing, as shown in Fig. 5.

The recorded X-ray images of DPF-1000U discharges also revealed the appearance of the so-called hot-spots (small plasma regions of a very intense X-ray emission), as it can be seen in Fig. 6.

Such a phenomenon was observed earlier in many PF experiments, e.g., in the MAJA-PF facility [6]. It could be explained to result from magnetohydrodynamic (MHD) instabilities of individual current filaments, particularly the $m=0$ ('sausage') and $m=1$ ('kink') instabilities, which can arise during the filament evolution [5]. From the collected experimental data one can deduce that 'hot-spots' appear often in the first shots after the venting of the PF experimental chamber or in shots with a small admixture of a heavy noble gas. In general, the presence of heavier gases or their mixtures (such as air), due to the higher $\mathrm{Z}$ of their ions, results in an increase in the X-ray emission when hot-spots become more intense.

In the reported experiments it was also observed that DPF-1000U shots with higher neutron yields demonstrate more dense fine structure, while those with medium yields show increased X-ray emission from a smaller plasma volume, as presented in Fig. 7.

Some shots in June 2013 session were performed with carbon-fibre composite (CFC) targets placed at a distance of $10 \mathrm{~cm}$ from the anode end-surface. The obtained X-ray images showed higher emission from a region situated near the CFC target surface, as shown in Fig. 8. This emission did not correspond to low-energy carbon lines, but it evidently originated from the identified region. This technique might be helpful in investigating how the plasma-target interaction occurs, and how does the presence of a target affect the process.

\section{Experimental results: time- and space-resolved measurements}

The PIN-diode signals recorded for each shot provided valuable data for further analysis and comparison
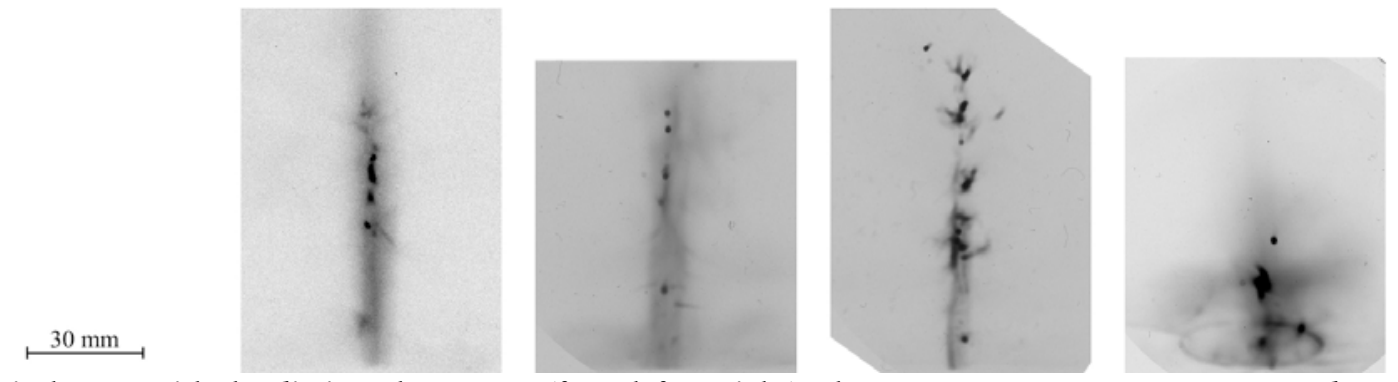

Fig. 6. Discharges with the distinct 'hot-spots' (from left to right): shots \#9987, \#10 143, \#10 144, and \#10 338.

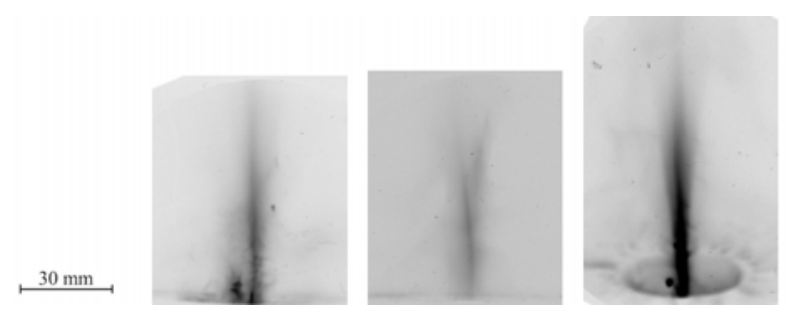

Fig. 7. X-ray images of discharges with different neutron yields: shot \#10220 (medium yield), shot \#10271 (low yield), and shot \#10 336 (high yield).
Fig. 8. X-ray images of shots with a visible CFC-target emission: shots \#10 002 and \#10 003. Dark spots at the top indicate an increased X-ray emission from the target material. 
Table 2. Sensitivities of different measuring channels during the recording of PIN-diode signals from the investigated DPF-1000U discharges

\begin{tabular}{lcccc}
\hline & Channel 1 & Channel 2 & Channel 4 & Channel 3 \\
\cline { 2 - 5 } & \multicolumn{5}{c}{ Sensitivity [mV/div] } \\
\hline 10074 & 300 & 100 & 100 & 100 \\
10080 & 400 & 200 & 100 & 100 \\
10187 & 20 & 20 & 20 & 20 \\
10235 & 300 & 100 & 300 & 300 \\
10333 & 30 & 10 & 30 & 30 \\
10338 & 20 & 10 & 20 & 20 \\
\hline
\end{tabular}

with time-integrated images. Information about the sensitivity of each PIN-diode channel for the selected shots is given in Table 2. Sensitivity of the measuring channel in the digital oscilloscope was adjusted to record the X-ray peaks in their entirety.

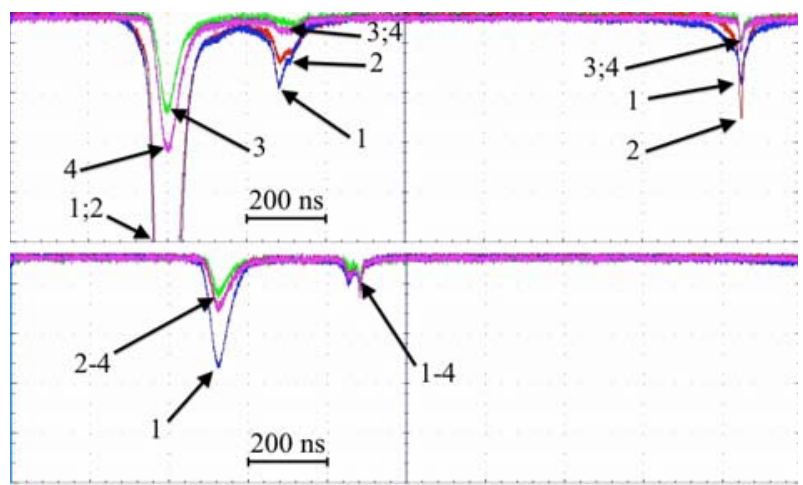

Fig. 9. PIN-diode signals recorded for shot \#10 074 (top) and \#10 080 (bottom). The waveforms correspond to channels presented in Fig. 1 and Table 2. The 'zero' position on the time scale was chosen as corresponding to the maximum of the first X-ray peak from channel 1.

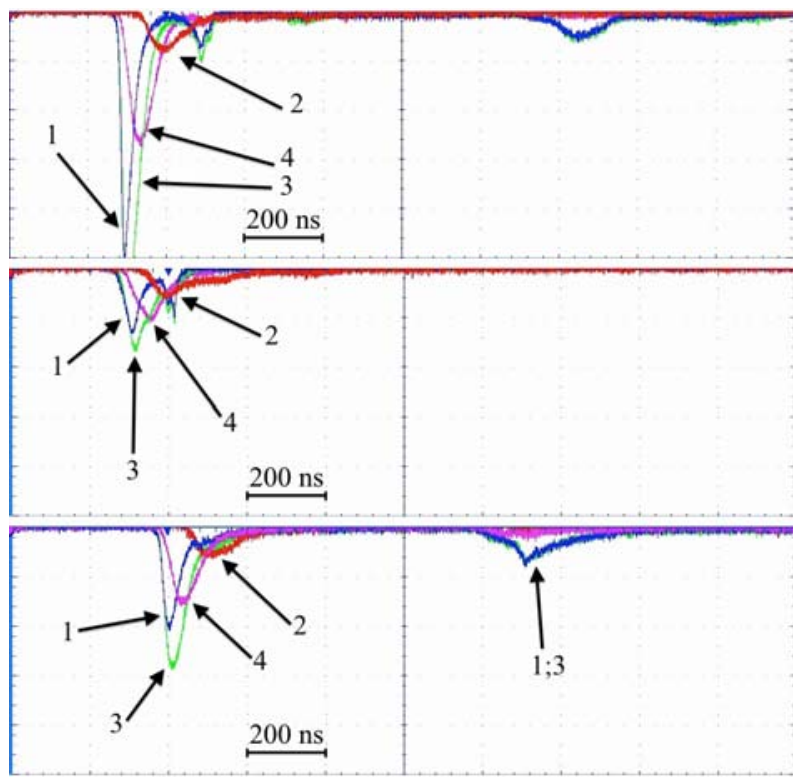

Fig. 10. PIN-diode signals obtained from shot \#10 187 (top), shot \#10 235 (middle), and shot \#10 333 (bottom). Blue (channel 1 observing a region $15 \mathrm{~mm}$ from the anode) and green (channel 3, $30 \mathrm{~mm}$ from the anode) peaks are very close, while magenta (channel $4,45 \mathrm{~mm}$ from the anode) and red (channel 2, $60 \mathrm{~mm}$ from the anode) are evidently shifted relative to each other. The 'zero' position on the time scale was chosen so as correspond to the maximum of the first X-ray peak from channel 1.
The collected data lead to the following conclusions: the DPF-1000U shots with neon as a working gas displayed the soft X-ray emission higher by about two orders of magnitude than those performed with the deuterium working gas. For shots observed with \#1 configuration of PIN diodes (various filters, each diode looking at a point $45 \mathrm{~mm}$ from the anode surface) the X-ray signals have often showed different peaks, as presented in Fig. 9. This might mean that each X-ray image was a result of two or more X-ray bursts occurring during the investigated discharge.

For shots observed with \#2 configuration of PIN diodes $(10 \mu \mathrm{m}$ Be filters each, different observation points) the recorded X-ray signals showed an evident time lapse between signals, as presented in Fig. 10. This means that the X-rays emitted from the regions observed at different distances were not emitted simultaneously.

This suggests that the X-ray emitting plasma region, which appeared at a larger distance from the anode outlet, was probably formed in a later phase of PF column compression. It should be noted that in some cases another X-ray pulse was recorded about $1 \mu \mathrm{s}$ after the first one. It was observed only in two measuring channels observing a region closer to the anode surface, as it can be seen in the images of shot \#10 333 (shown in Fig. 5) and shot \#10 187 (shown in Fig. 11). Such a signal might be a result of some instabilities which appear mostly during the later phase of the PF discharge.

The X-ray signals obtained from shot \#10 338 (shown in Fig. 12) might be easily compared with the X-ray integrated image (shown earlier in Fig. 6). Such a comparison suggests that isolated hot-spots are formed independently at different instants of the discharge.

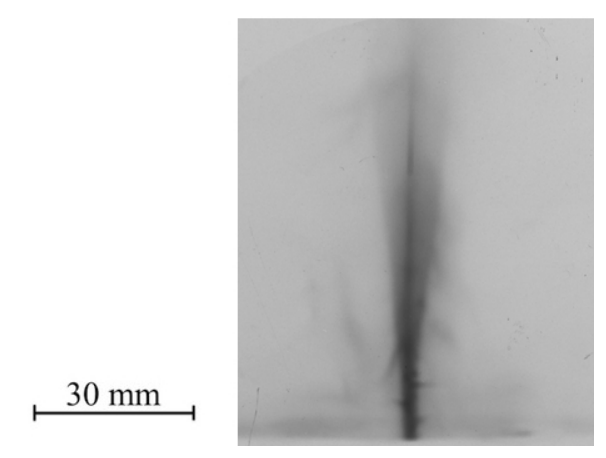

Fig. 11. X-ray image of shot \#10 187. Distinct filaments can easily be seen. Intense X-ray emission from the region close to the anode corresponds to two distinct X-ray pulses (shown previously in Fig. 10). 


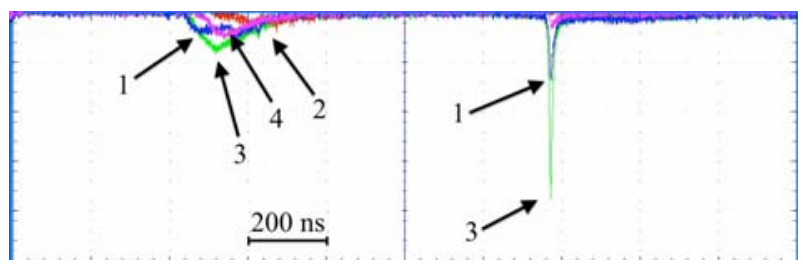

Fig. 12. X-ray signals from shot \#10 338. A relatively weak and extended in time pulse was followed by a strong pulse. The X-ray integrated image of this shot (presented in Fig. 6) showed a very strong emitting region exactly in the place which was observed by the channel 3 - PIN diode. That hot-spot was formed probably $1 \mu$ s later than other structures in the investigated discharge. The 'zero' position on the time scale was chosen so as to correspond to the maximum of the first X-ray peak from channel 1.

\section{Summary and conclusions}

The reported DPF-1000U experiments confirmed the appearance of filamentary structures in plasma-focus discharges as well as the existence of the correlation between a high neutron yield and dense symmetric discharge structure. It was observed that the intense soft X-ray emission did not coincide with a high neutron yield, while the gas-puffing had evidently a stabilizing influence.

More PF shots are needed - particularly discharges with an admixture of heavy noble gases as well as space- and time-resolved measurements of X-ray emission - in order to formulate stronger conclusions about the fine-structure of plasma-focus discharges. In particular, simultaneous time- and space-resolved measurements (with a better resolution) should be performed to examine a time lapse between the X-ray emission from plasma regions placed at various distances from the anode end.
Acknowledgment. Work was performed at NCBJ in Swierk and IPPLM in Warsaw, Poland. This work was partly supported by the IAEA CRP RC-16954 and RC16956 grants as well by the Polish Ministry of Science and Higher Education within the framework of the financial resources in the year 2014 allocated for the realization of the international co-financed projects.

\section{References}

1. Lebert, R., Neff, W., \& Rothweiler, D. (1996). Pinch plasma source for X-ray microscopy with nanosecond exposure time. X-Ray Sci. Technol., 6, 107-140. DOI: 10.3233/XST-1996-6201.

2. Bernard, A., Bruzzone, H., Choi, P., Chuaqui, H., Gribkov, V., Herrera, J., Hirano, K., Krejci, A., Lee, S., Luo, C., Mezzetti, F., Sadowski, M. J., Schmidt, H., Ware, K., Wong, C. S., \& Zoita, V. (1998). Scientific status of plasma-focus research. J. Moscow Phys. Soc., 8, 93-170.

3. Sadowski, M., Herold, H., Schmidt, H., \& Shakhatre, M. (1984). Filamentary structure of the pinch column in plasma focus discharges. Phys. Lett. A, 105, 117-123. DOI: 10.1016/0375-9601(84)90650-9.

4. Scholz, M., Drozdowicz, K., Karpinski, L., Woźnicka, U., Krauz, V., Kubes, P., Paduch, M., Sadowski, M. J., Czaus, K., Malinowski, K., Kwiatkowski, R., Skladnik-Sadowska, E., \& Zebrowski, J. (2013). Status of plasma-focus research in Poland. In Proceedings of International Conference PLASMA-2013, September 2-6, 2013 (T10). Warsaw, Poland. http://plasma2013. ipplm.pl/.

5. Sadowski, M. J., \& Scholz, M. (2008). The main issues of research on dense magnetised plasmas in PF discharges. Plasma Sources Sci. Techn., 17, 024001. DOI: 10.1088/0963-0252/17/2/024001.

6. Jakubowski, L., Sadowski, M., Baronova, E. O., \& Vikhrev, V. V. (1997). Study of X-ray polarization and e-beams generated during hot-spots formation in PF-discharges. Dense Z-pinches. AIP Conf. Proc., 409, 443-447. DOI: 10.1063/1.53825. 